\title{
THE LIMITATIONS OF STATISTICAL LOW RUNS PREDICTION IN ROUGH SEAS: A STUDY BASED ON REAL WAVE DATA
}

\author{
G. Caiazzo ${ }^{1}$, D.J. Taunton ${ }^{1}$, P.A. Wilson ${ }^{1}$ \\ ${ }^{1}$ University of Southampton, Boldrewood Innovation Campus, Burgess Rd, Southampton SO16 7QF, UK.
}

\begin{abstract}
This work aims to estimate the feasibility of wave runs statistical prediction in rough seas, with a specific application of Kimura's theory (Kimura, A.,1980). According to Kimura, the probability of a low run of waves of assigned length is a function of the correlation between consecutive waves in a group. Time series from wave buoy measurements (located at Bideford Bay, England and data downloaded from the Channel Coast website) have been tested. The spectral analysis showed that higher values of the significant wave height correspond to broader bandwidth spectra. The Kimura's low runs prediction is noticeably affected by this aspect: by plotting the low runs probability against the number of incoming waves, narrow spectra show a steeper behaviour of the low runs probability curve, whereas broader spectra correspond to flat probability curves settled on lower values. Therefore, it appears that broader spectra are characterised by more grouping phenomena. Moreover, the Kimura's estimation has been calculated in an adaptive frame applied to the signal, in order to test the sensitivity to the length of the data; a minimum length of 6 to 8 hours has been found to have a stable prediction of the low runs probability.
\end{abstract}

Keywords: Quiescent Period Prediction; wave runs prediction; signal processing.

\section{Introduction}

The problem of predicting the evolution of the sea state is an important topic in several operational marine scenarios. A relevant example is the problem of predicting the Quiescent Periods (QP) for launch and recovery of vehicles from ships in rough seas: in general, a QP is defined as the amount of time during which the ship motion or encountered wave amplitude stays below assigned thresholds, depending on the specific application or operation. The success of such an operation entirely relies on the accuracy of the prediction of the surface behaviour in the very short term (in ranges between 30 seconds to 5 minutes), as a fast prediction of the wave elevation allows an immediate estimation of the resulting ship motion. It is clear how such accurate results can be crucial in high risk operations, during which for example the pilot of an air vehicle does not have a clear visual sense of the vertical movement of the ship, therefore the importance of a landing-aid technology is vital. Besides the realm of vehicle launch and recovery, the problem of short time ship motion prediction is important in regard to the future of unmanned naval transport. EU's FP7 project MUNIN aims to develop a concept for an autonomous dry bulk carrier, as highlighted by Burmesteir et al. [1]. However, a scenario of unmanned ferries for civil transport appears to be an established reality in the future, Levander [2]: it is expected that such vessels will be equipped with a QP 
estimator in case of accidents or emergency situation, in order to allow the passengers to leave the ship in the most favourable sea conditions possible.

The literature makes a general distinction between two essential approaches to the topic of sea surface elevation prediction. Usually the methodologies that rely on statistical elaboration of 'previous' sea elevation data in order to estimate probabilities of a run of either low or high waves are considered as 'backwards'; a 'run' is meant as a series of consecutive waves exceeding or being inferior to a specified threshold (hence the definition of high or low run). Vice versa, methodologies that implement wave propagation models based on the current surface elevation measurements are typically defined as 'forwards', as they do not rely on the wave time history. A comprehensive overview of both deterministic prediction methodologies and the traditional statistics is illustrated by Giron-Sierra et al [3]. In regard to the topic of wave data acquisition in real time by using X-radar technology, Nieto-Borge [4] describes a technique to estimate wave elevation maps which takes into account a modulation transfer function to describe the radar imaging mechanisms at grazing incidence and horizontal polarization.

One of the most renowned backwards run prediction methodology is Kimura's theory, Kimura [5]: as it will be largely explained in section 4, Kimura's theory schematizes the evolution of the sea surface as a Markov chain, according to which the transition from a state to the next one is only a function of the immediately previous state. In this regard, the prediction of a run is based on the wave to wave correlation factor. Kimura assumes that the probability of two consecutive waves exceeding or staying below an arbitrary threshold can be represented by a Rayleigh distribution, which is a function of the aforementioned wave to wave correlation parameter. Kimura's runs prediction theory has not been traditionally used in the realm of ship motion prediction, although it finds large application in the problem related to the stress fatigue prediction on wave energy converters (WEC) due to the impact of consecutive waves exceeding an assigned critical threshold. Coastal defence is another context of frequent use of the Kimura's methodology. In this regard, the literature mostly deals with the problem of predicting the length of high runs from a condition of a quiet sea, with particular regard to applications such as coastal defence and the aforementioned energy wave converters structural stability or harvested energy prediction. However, many other applications require to approach the problem from the opposite perspective: launch and recovery of vehicles on ships in rough seas is a remarkable case where the prediction of a quiescent period of the vessel is crucial to perform the operation in safety.

In this paper, the authors aim to test the applicability of the traditionally well-known Kimura's theory for determining QP prediction for maritime launch and recovery operations.

A first problem consists in the choice of the data to test, as in several cases wave records in specific conditions (for examples wave measurements in open sea) are not easily available or, more generally, it is difficult to access an arbitrarily long record of wave data corresponding to the conditions of interest. In this regard, a very common approach in the realm of runs prediction is to assume the sea state represented by a standard ideal spectrum, hence it is possible to generate a synthesized wave time history from the frequency domain according 
to the target spectrum. However, the literature shows examples where both synthetic data and long wave records acquired from the field have been both used and compared: Elgar et al. [6] compare the low runs probability from the Kimura's model for both synthetic waves and from a real wave record measured with bottom-mounted pressure sensors, which continuously gathered data for 5 hours daily over a period of 4 weeks. A comparison between the mean length of the runs calculated on both theoretical and experimental waves shows good agreement, although the authors do not specify the conditions of the sea state during the acquisition.

The work of Saulnier et. al [7] implicitly confirms the reliability of the Kimura's model in such contexts: the paper focuses on the energy harvesting prediction for a WEC stochastic model which is tested with both buoy data (two wave time series collected on a period of 13 and 9 years respectively) and with theoretical waves. As the harvested energy is proven to be a function of peak period, significant wave height and wave groupiness, good consistency between the results from synthetic and real data can be considered as a good estimator for the Kimura's theory performed on long time series.

It is evident how such literature always considers technical cases where the analysed data (both synthesized and real) have been gathered over long periods, or at least cases where a long sampling time is possible. Weeks or even months of recorded or simulated data can be a standard time on which Kimura's theory is applied. Moreover, most of the data used in such works correspond to conditions of calm sea or, in general, a sea state that is far from a rough sea scenario. However, to the knowledge of the authors, there are apparently no works showing the application of Kimura's theory to wave histories of rough seas, neither have significant studies been made on the reliability of the Kimura's theory in the short term. In this regard, the most relevant point to investigate is how the length of the recorded data affects the reliability of Kimura's results. Is there a minimum sampling time threshold and is it compatible with the short time operations involved in cases like landing and recovery of vehicles?

Generally, the literature approaching the problem of QP prediction in rough seas is small. An extensive presentation of the state of the art in deterministic sea wave prediction is given by Giron-Sierra [8], where the general principle of real time X-band radar data retrieval is explained and its subsequent implementation in a wave propagation model. Similarly, the process described by Giron-Sierra is well detailed in the work by Edgar et al.[9] , in which the maximum prediction time is estimated against a number of significant parameters, mainly water depth and spectral width of the analysed wave signal.

Although [8-9] seem to concur about the use of wave data recorded in real time as the standard for short time predictions, it is difficult to identify a standard methodology for the wave analysis process and the prediction of the surface elevation.

In light of these considerations, the work presented in this paper essentially focuses on two main points: firstly, the correlation between the weather conditions, the bandwidth of the spectrum of the wave time series and the corresponding wave to wave correlation parameter. 
Secondly, the sensitivity of the Kimura's method to the length of analysed data is considered: here, wave buoy measurements are tested by applying an adaptive frame analysis, as it will be shown in section 5 .

\section{Broadness factor and low runs probability according to Kimura}

The broadness factor $\varepsilon$ is a non-dimensional parameter which is generally used to estimate the 'narrow bandedness' of a wave time history and its wave energy spectrum, as described by Lloyd [10]. If $\xi(t)$ is the wave signal in the time domain, the Fourier transform 'translates' the signal to the frequency domain. The aim is to have a representation of how the energy of the signal is distributed at various frequencies; this quantity is known as a power spectral density (PSD). PSD is theoretically defined as:

$S(\omega)=\lim _{T \rightarrow \infty} E\left[|\hat{\xi}(\omega)|^{2}\right]$

$\hat{\xi}(\omega)$ is defined as:

$\hat{\xi}(\omega)=\frac{1}{\sqrt{T}} \int_{0}^{T} \xi(t) e^{-i \omega t} d t$

$E\left[|\hat{\xi}(\omega)|^{2}\right]$ represents the expected value.

In this work, $S(\omega)$ has been calculated by implementing the Fast Fourier Transform algorithm (FFT) in Matlab.

The broadness factor definition, according to Lloyd [10], Cartwright [11] and Longuet - Higgins [12] , can be calculated from the PSD though the spectral momenta $M_{n}$ :

$\varepsilon=\sqrt{1-\frac{M_{2}^{2}}{M_{0} \times M_{4}}}$

where $M_{0}, M_{2}$ and $M_{4}$ represent the $0^{\text {th }}, 2^{\text {nd }}$ and $4^{\text {th }}$ order momenta, defined by the generic $n^{\text {th }}$ order equation:

$M_{n}=\int_{0}^{\infty} S(\omega) \times \omega^{n} d \omega$

$\varepsilon$ has value between 0 and 1: values close to 0 represent a narrow spectrum, whereas a wide band spectrum corresponds to a broadness factor tending to 1 .

Theoretically, in the simplified hypothesis to approximate a pure swell sea as a simple sine wave, its broadness factor equals to zero, as the spectrum calculated for such types of wave signal reduces to only one frequency component. A more realistic representation of a swell sea includes more frequency components and a more complex sea state, for example a 'rough' sea condition, is expected to have a richer spectrum and therefore a higher value of the broadness factor.

The Kimura's theory uses an estimation of the wave to wave correlation in order to estimate the probability of a high or low run and its corresponding time length. Therefore, it is 
important to investigate how the sea conditions affect the broadness factor $\varepsilon$ and the estimation of the low runs probability according to Kimura's theory.

More specifically, Kimura's runs prediction model estimates the probability of a run by considering the sea state as a Markov chain system, which considers the probability of transition to the next state to be solely dependent upon the previous state and not the entire history. Kimura uses a two dimensional Rayleigh distribution to describe the probability of two consecutive wave heights $h_{1}$ and $h_{2}$. This probability value is expressed by LonguetHiggins [12] as:

$$
p\left(h_{1}, h_{2}\right)=\frac{h_{1} h_{2}}{M_{0}^{2}\left(1-k^{2}\right)}\left(e^{-\left(h_{1}^{2}+h_{2}^{2}\right) / 2 M_{0}\left(1-k^{2}\right)}\right) I_{0}\left(\frac{k}{1-k^{2}} \frac{h_{1} h_{2}}{M_{0}}\right)
$$

where:

$$
k=\frac{\left(X^{2}+Y^{2}\right)^{1 / 2}}{M_{0}}
$$

$$
\begin{aligned}
& X=\int_{0}^{\infty} S(\omega) \cos (\omega-\bar{\omega}) \tau d \omega \\
& Y=\int_{0}^{\infty} S(\omega) \sin (\omega-\bar{\omega}) \tau d \omega
\end{aligned}
$$

where

$$
\bar{\omega}=\frac{M_{1}}{M_{0}}
$$

and

$$
\tau=\frac{2 \pi}{\bar{\omega}}
$$


Io represents the modified Bessel function of order 0 ; the wave correlation coefficient $\gamma$ is defined as:

$\gamma=\frac{1}{\sigma^{2}(H)} \frac{1}{N-1} \sum_{i=1}^{N-1}\left(H_{i}-H_{m}\right)\left(H_{i+1}-H_{m}\right)$

with $\sigma(H)$ is the standard deviation of a large number $N$, of wave heights $H$, whereas $H_{m}$ representsthe mean wave height. As defined by Uhlenbeck [13], $k$ is correlated to the wave to wave correlation coefficient $\gamma$ according with the following equation:

$\gamma=\frac{\mathbf{E}(k)-\frac{1}{2}\left(1-k^{2}\right) \mathbf{K}(k)-\frac{\pi}{4}}{1-\frac{\pi}{4}}$

where $K$ and $E$ represent complete elliptic integrals of the first and second kind respectively.

However, according to Cartwright and Longuet-Higgins [11], for broad spectra (corresponding to $k \simeq 1$ ), it is possible to prove that :

$$
\gamma \sim 1-\left(1-k^{2}\right) /(4-\pi)
$$

For narrower spectra, when $k^{2}$ is less than 0.6, according to Longuet - Higgins [12] it can be assumed that:

$\gamma \sim k^{2}$

The probability that a certain wave exceeds or is lower than a critical threshold $h^{*}$ can be expressed as:

$$
\begin{aligned}
& p\left(H_{j}\right)=p_{+}^{j-1}\left(1-p_{+}\right) \\
& p\left(L_{j}\right)=p_{-}^{j-1}\left(1-p_{-}\right)
\end{aligned}
$$

$p\left(H_{j}\right)$ and $p\left(L_{j}\right)$ express the probability of a high and low runs respectively, whose length is equal to the number of waves $j$. The conditional probabilities $p_{+}$and $p$-can be calculated directly from the joint density expressed in equation (5).

$$
p_{+}=\int_{h^{*}}^{\infty} \int_{h^{*}}^{\infty} p\left(h_{1}, h_{2}\right) d h_{1} d h_{2} / \int_{0}^{\infty} \int_{h^{*}}^{\infty} p\left(h_{1}, h_{2}\right) d h_{1} d h_{2}
$$




$$
p_{-}=\int_{0}^{h^{*}} \int_{0}^{h^{*}} p\left(h_{1}, h_{2}\right) d h_{1} d h / \int_{0}^{\infty} \int_{0}^{h^{*}} p\left(h_{1}, h_{2}\right) d h_{1} d h_{2}
$$

If $p\left(H_{j}\right)$ and $p\left(L_{j}\right)$ represent the probability of a run of $j$ consecutive waves, it is possible to calculate the corresponding mean length of the run as:

$\bar{H}=\sum_{1}^{\infty} p\left(H_{j}\right)=\frac{1}{1-p_{+}}$

$\bar{L}=\sum_{1}^{\infty} p\left(L_{j}\right)=\frac{1}{1-p_{-}}$

At this point, it is interesting to investigate a possible correlation between $\varepsilon$ and $\gamma$ and its consequences on the low runs prediction expressed by equation 16.b. As the wave to wave correlation parameter can be considered as an indicative factor about the possibility of grouping phenomena in a wave time series, it is important to evaluate its possible correlation with the spectral width of the original signal and therefore with the low runs prediction. In the next section, both $\varepsilon$ and $\gamma$ have been evaluated for the Channel Coast data, extracted from different months throughout the year; moreover, the significant wave height for such signals has been compared to the broadness factor, in order to test the dependence of the spectral width from the weather conditions.

\section{3. $\varepsilon$ and $\gamma$ for wave buoy data}

In this section we compare the broadness factor $\varepsilon$ and the wave to wave correlation parameter $\gamma$ for the wave buoy data from the Channel Coast website [14].

More specifically, the sampling point is Bideford Bay, England, Fig 1, where several time series of 24 hours in length, sampled at a frequency $F_{s}=1.28 \mathrm{~Hz}$ and ranging from April 2017 to April 2018 have been considered. 


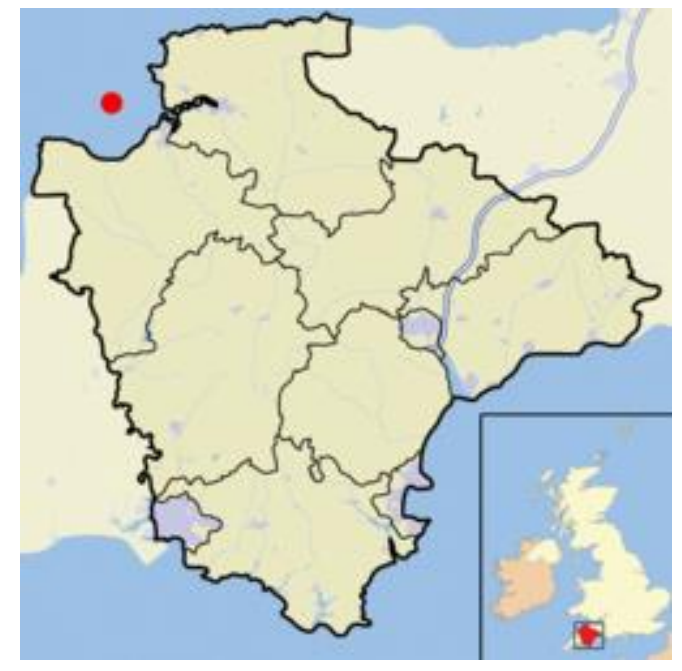

Figure 1-Bideford Bay

Fig.2 reports the behaviour of the significant wave height $\bar{H}_{1 / 3}$ for each time series of the Channel Coast data recorded at Bideford Bay, corresponding to each month from May 2017 to April 2018, in intervals of 30 minutes. $\bar{H}_{1 / 3}$ has been calculated according with Cartwright and Longuet-Higgins [11][12] from the spectral momentum $M_{o}$ defined by equation (4) and the broadness factor $\varepsilon$ defined by equation (3):

$\bar{H}_{1 / 3}=4.00 \sqrt{M_{0}} \sqrt{1-\frac{\varepsilon^{2}}{2}}$ 

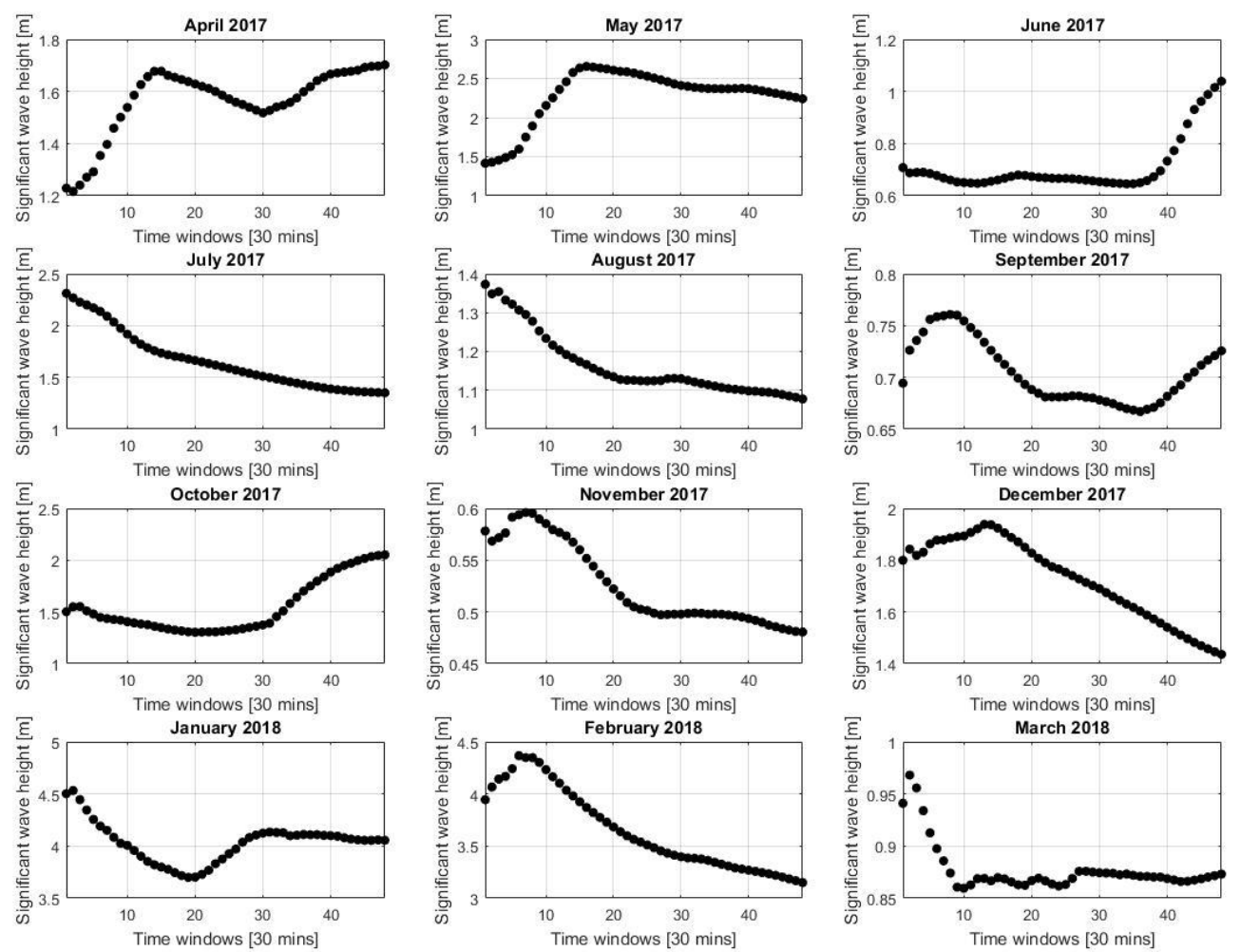

Figure 2 - Significant wave height of the Channel Coast wave buoy measurements

Fig.3 shows the broadness factor calculated for the Channel Coast data: as previously mentioned, each time series is 24 hours long. The signal has been analysed in adjacent windows of 30 minutes, within which the broadness factor has been calculated. 

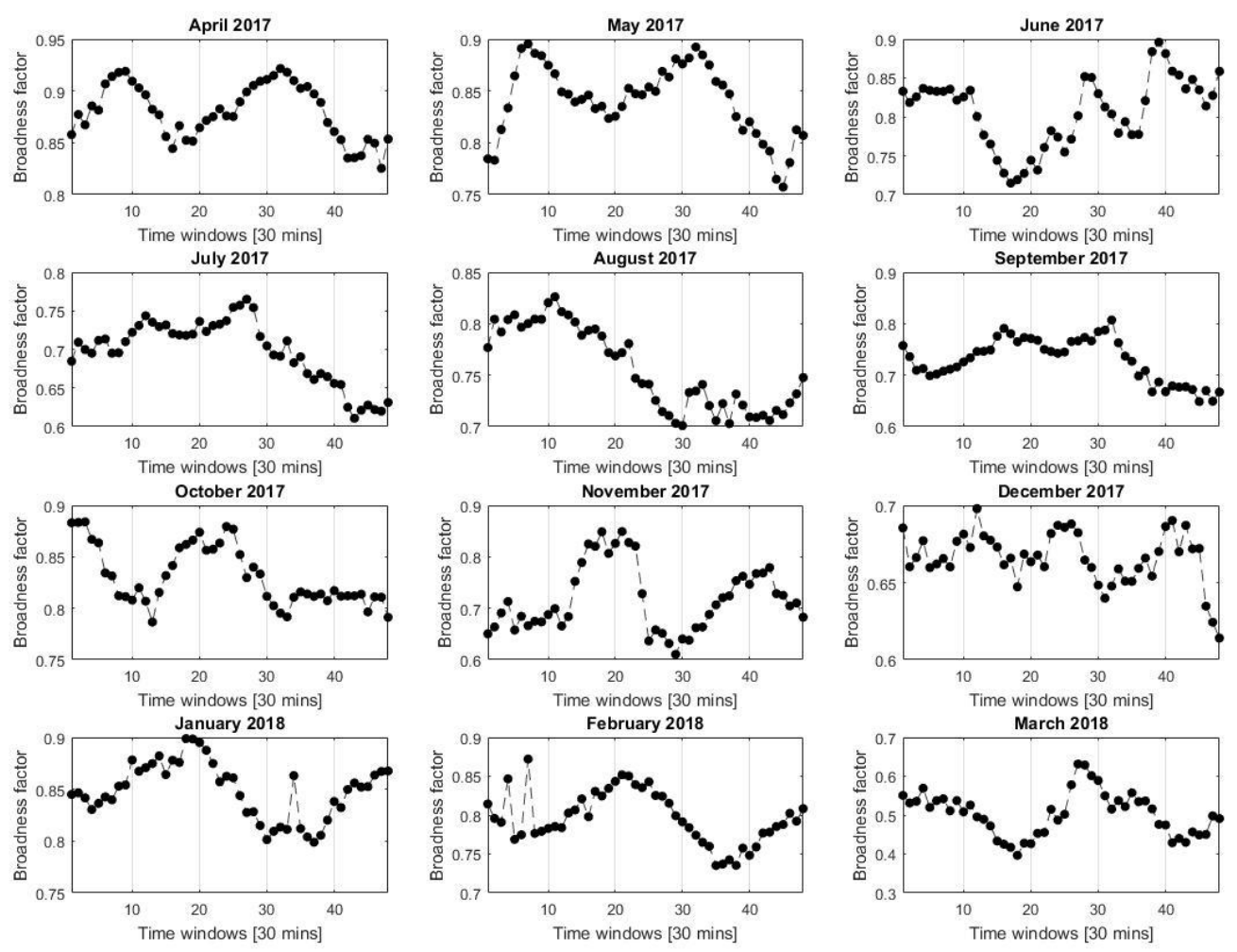

Figure 3.a-Broadness factor for Channel Coast wave data 

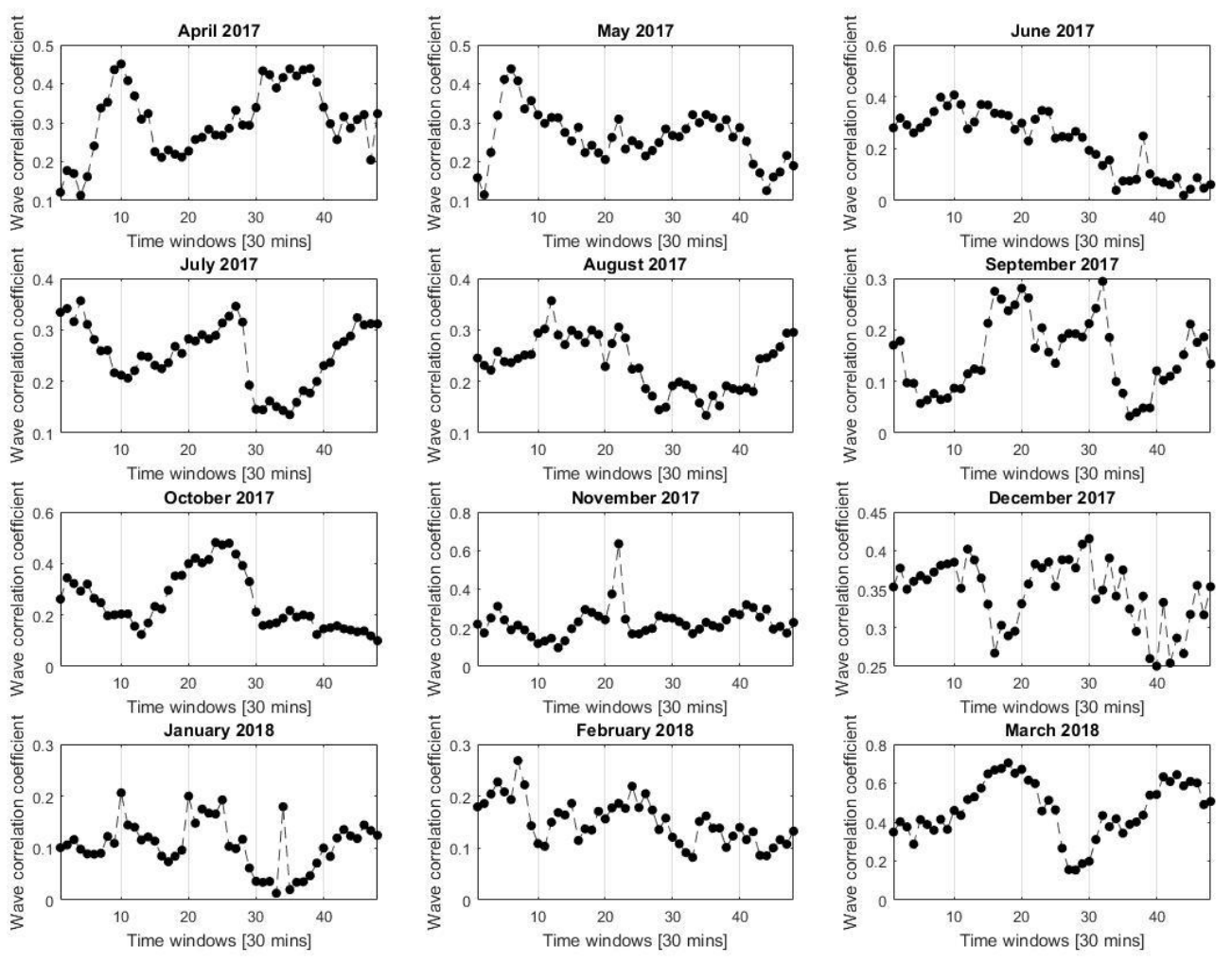

Figure 3.b - Wave correlation coefficient for Channel Coast wave data 

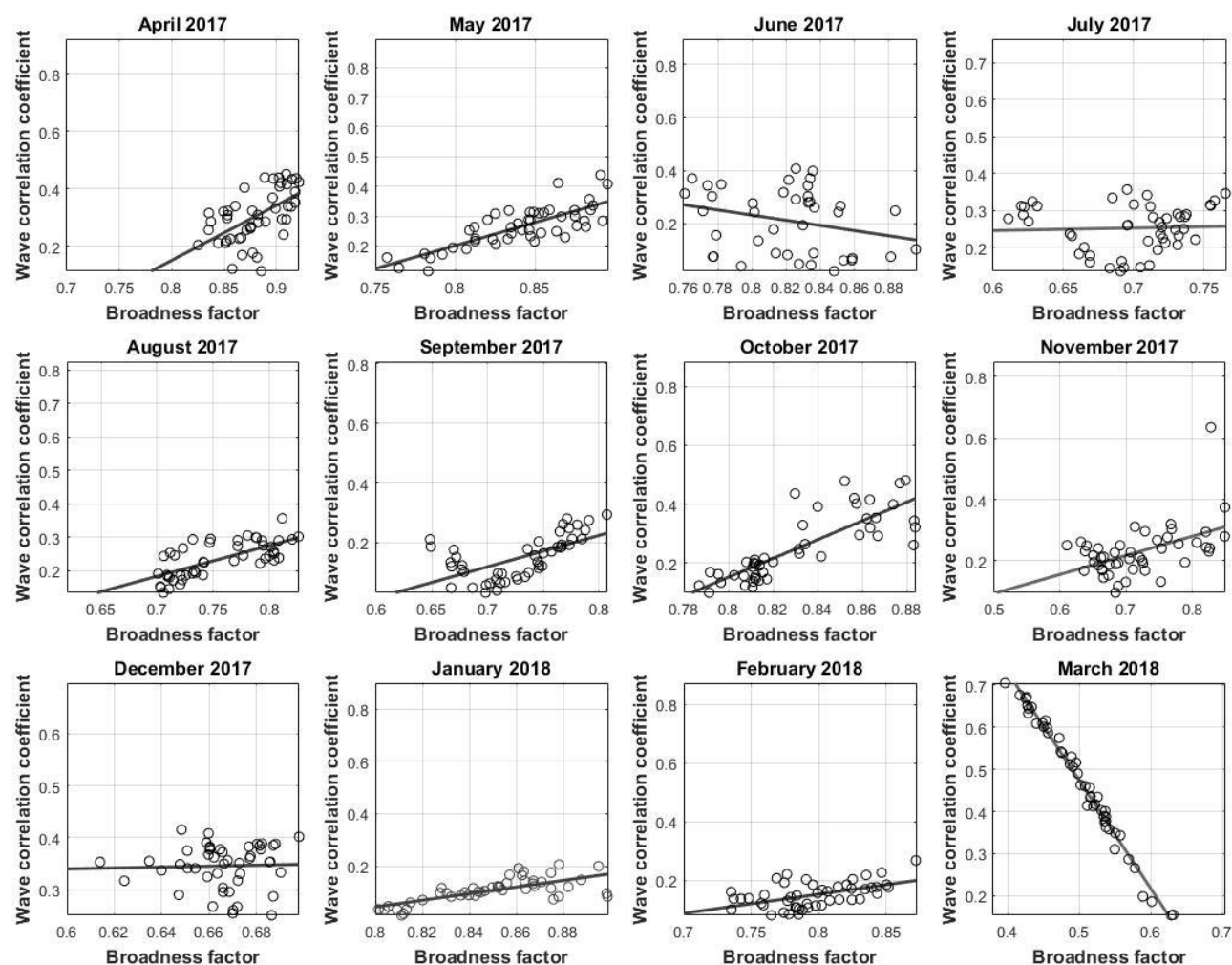

Figure 2.c - Linear regression of broadness factor and wave correlation coefficient for the Channel Coast data 

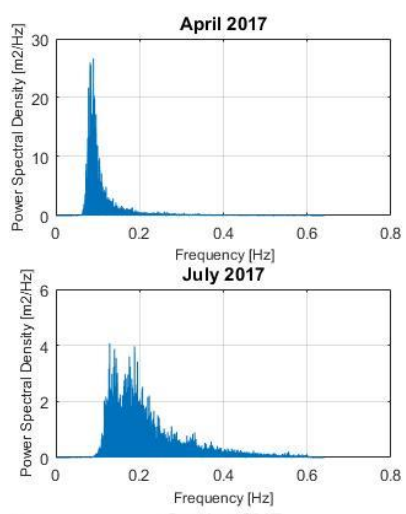

October 2017

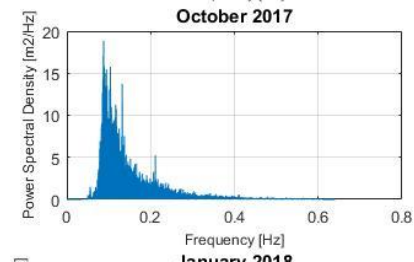

January 2018

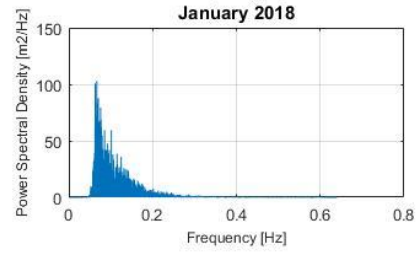

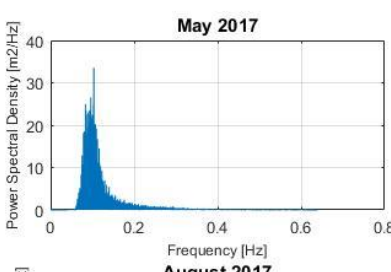

August 2017

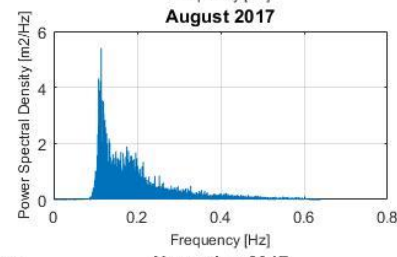

November 2017

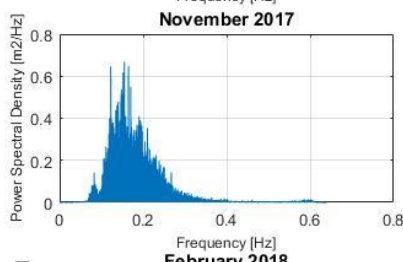

February 2018

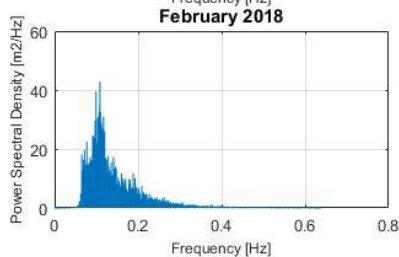

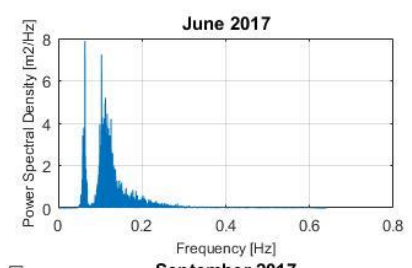

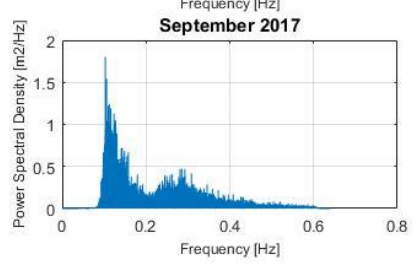

December 2017

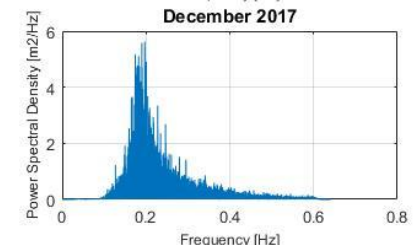

March 2018

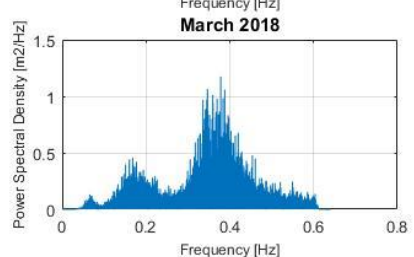

Figure 3.d-Power spectral density of the Channel Coast time series

Fig. 3.d reports the power spectral density calculated for the Channel Coast time series: it is important to consider these diagrams and observe the distribution of the frequencies along the spectrum. According with (3), the broadness factor depends on M4, which is noticeably affected by the truncation error of the original data. In Fig.3.d it is possible to see that the effect of the truncation error can be neglected in all the analysed time series.

Fig.3.a,b show the broadness factor and the wave correlation coefficient of the analysed wave buoy time series respectively; one a time span corresponding to one year, $\varepsilon$ ranges in a broad interval from around 0.5 to 0.9 , whereas $\gamma$ ranges in an even broader interval from 0.1 to 0.9 . Fig. 3.c shows the linear regression between $\varepsilon$ and $\gamma$ for the time series corresponding to each month: it is possible to notice that, generally, an increment of the broadness factor corresponds to and increment of the wave correlation coefficient. In some cases (April 2017, October 2017, January 2018), increments of $\gamma$ can be observed on a very narrow range of $\varepsilon$; in July 2017, and December 2017 it is possible to observe how $\gamma$ appears to stay constant. It is important to compare this results with the corresponding sea conditions

A comparison of the significant wave height data (Fig.2) with the Douglas scale [15] shows that the series from January 2018 and February 2018 correspond to a condition of very rough sea (average maximum wave height equal to $5 \mathrm{~m}$ ), whereas the data from November 2017 and March 2018 correspond to a condition of calm sea. It is possible to observe that the time series corresponding to January 2018 shows a value of the broadness factor floating around 
0.85 , whereas the time series corresponding to March has a value of $\varepsilon$ oscillating between 0.4 and 0.55 .

\begin{tabular}{|c|l|l|l|}
\hline Degree & \multicolumn{1}{|c|}{ Height [m] } & Height [ft] & \multicolumn{1}{|c|}{ Description } \\
\hline 0 & \multicolumn{2}{|c|}{ No wave } & Calm (glassy) \\
\hline 1 & $0-0.10$ & $0.00-0.33$ & Calm (rippled) \\
\hline 2 & $0.10-0.50$ & $0.33-1.64$ & Smooth \\
\hline 3 & $0.50-1.25$ & $1.6-4.1$ & Slight \\
\hline 5 & $1.25-2.50$ & $4.1-8.2$ & Moderate \\
\hline 6 & $2.50-4.00$ & $8.2-13.1$ & Rough \\
\hline 7 & $6.00-6.00$ & $13.1-19.7$ & Very rough \\
\hline 8 & $9.00-14.00$ & $29.5-45.9$ & Very high \\
\hline 9 & $14.00+$ & $45.9+$ & Phenomenal \\
\hline 5.00 & $19.7-29.5$ & High \\
\hline
\end{tabular}

Generally, it appears that for the buoy data corresponding to calm sea conditions, $\varepsilon$ ranges in an interval of lower values than those corresponding to rough weather, where the time series appear to be characterized by narrow ranges of high broadness. Although the wave correlation coefficient $\gamma$ generally increases with the broadness factor, conditions of rough weather do not seem to correspond necessarily to an increase of $\gamma$. Therefore, whereas a time series with a broad spectrum generally corresponds to an higher correlation between successive waves, the grouping phenomena occurring in a time series are not necessarily a consequence of the weather conditions. 


\section{Kimura's low run prediction}

In Fig.5, the low run probability curves corresponding to each wave buoy time series is reported. The probability is plotted against the number of incoming waves: this diagram simply expresses the probability of a low run of an assigned length, which is expressed as the number of incoming waves in the range from 1 to 8 . The longer the assigned time length, the lower the probability of a run of that length. It is possible to observe how the curves broadly range from a flatter shape which is settled on lower probability values, to steeper behaviour which exhibit significantly higher probabilities for short time lengths (e.g. for 'fewer' incoming waves) which rapidly decreases as the number of waves increases.

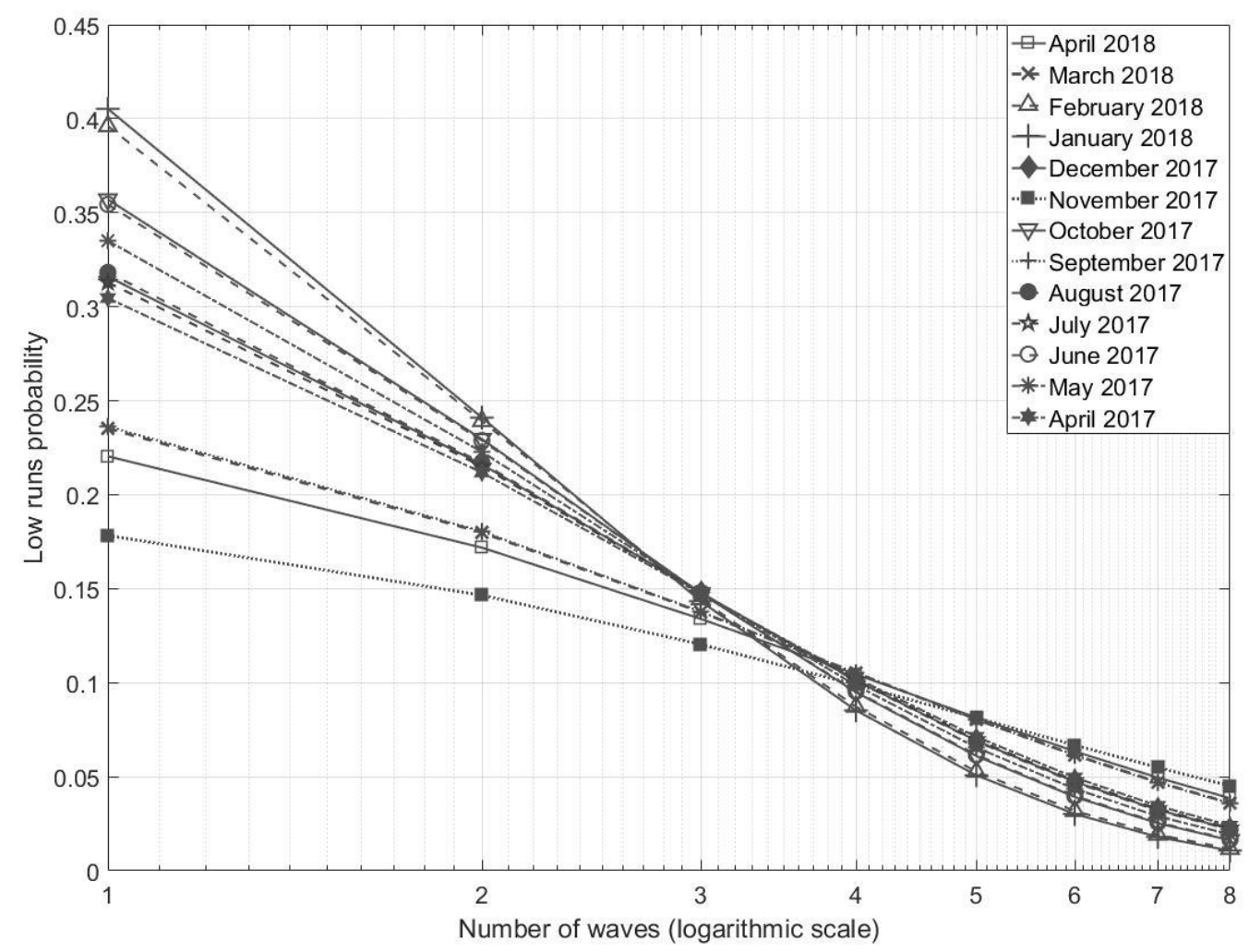

Figure 4-Low runs prediction for time series extrapolated monthly from April 2017 to April 2018

In order to understand the reason of such different behaviour according with the period of the year during which the time series has been measured, an evaluation of the significant wave height for each time series has been calculated. Fig.5 shows the values of both maximum as significant wave height in June and November 2017, which respectively correspond to a flat and a steep probability behaviour in Fig.4. 

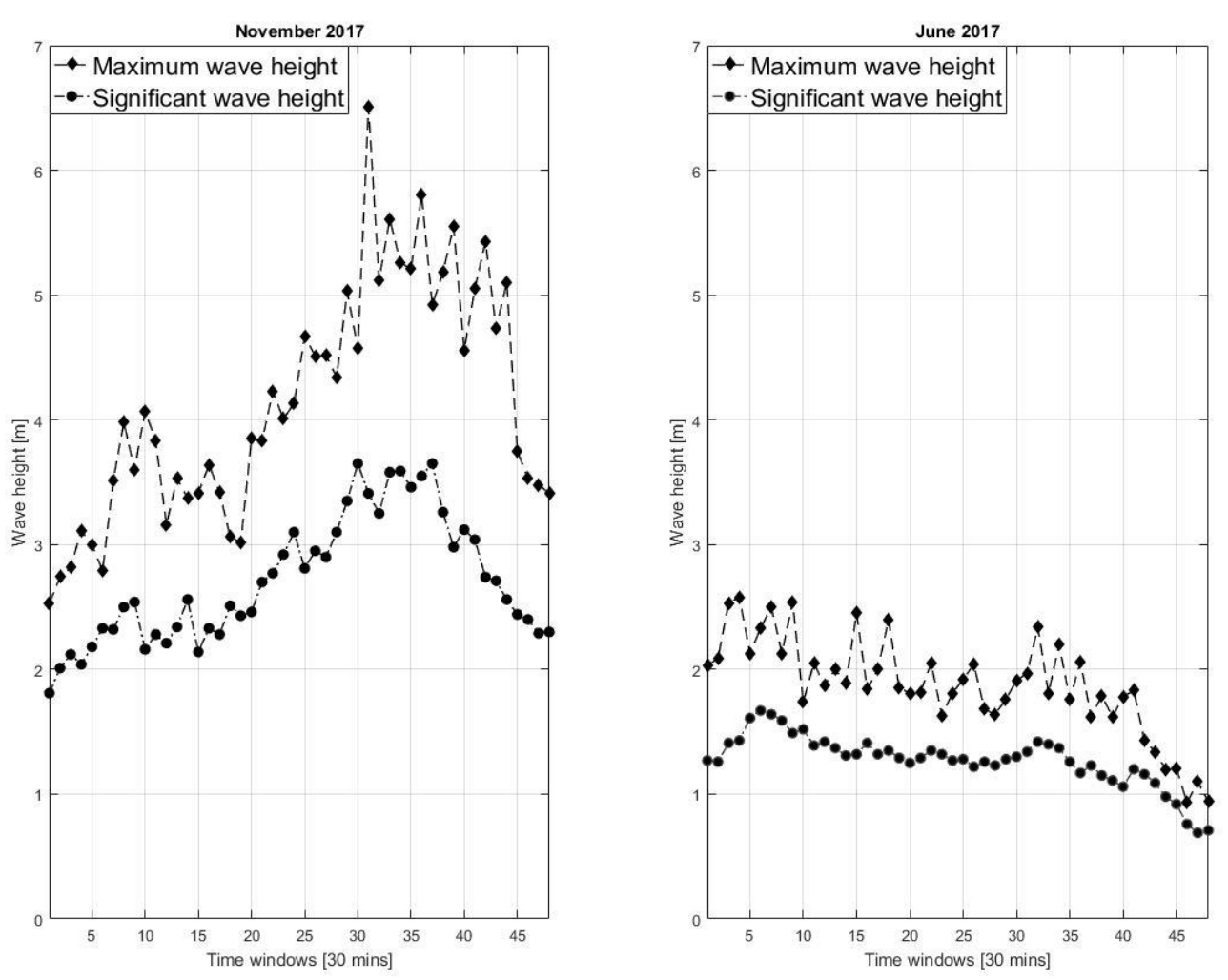

Figure 5 - Maximum and significant wave heigth for Channel Coast buoy data

It is to expected that the June 2017 series has a lower significant and maximum wave height than the November 2017 series.

This result seems to be confirmed also in the low runs prediction obtained from the Kimura's theory. Waves in rough seas are more statistically correlated and therefore characterized by higher groupiness effects; and vice versa, waves in calm seas are less correlated and they are characterized by less grouping phenomena. This result can also be confirmed in the wave correlation coefficient diagrams in Fig.3.b.

From the application of the Kimura's methodology to the Channel Coast data, it appears that the time series corresponding to rough weather conditions show a flat behaviour and a general low value of the low runs probability. Such values range between 0.1 and 0.25: in a real navigation scenario characterized by rough weather conditions, it is clear that the success of a delicate operation (like landing or recovery of a vehicle) completely relies on the accuracy of the prediction of the sea surface elevation. The low runs probability values obtained from the Kimura's methodology applied to the buoy wave data appear to be incompatible with the accuracy that is requested in sensitive marine operations. 


\section{Kimura's low run prediction applied in an adaptive frame context}

As mentioned in the previous section, the issue about applying a statistics based methodology for the wave elevation prediction is the amount of data needed in order to have a reliable result. As clarified in section 4 , the spectrum of a wave record sample, especially in the case of rough water, is unlikely to be represented by an ideal spectrum: for this reason, it is expected that the spectral shape changes from sample to sample. In order to verify this assumption, wave buoy time series have been analysed in an adaptive time window: specifically, a progressively increasing time window is applied to the signal and the Kimura's low runs probability is calculated at each, ever increasing window. The use of the adaptive window is used to find a threshold amount of data corresponding to the minimum sampling time required to have a stable prediction of the low runs: it will be shown that below the threshold, the estimation of the probability can oscillate before settling on a stable value.

Fig.6 summarizes the analysis iterative scheme: $w_{\text {SIZE }}$ is the incremental size of the window applied to the signal, resulting in an analysis frame which increases at each iteration $\mathrm{n}=$ $[1, \ldots, N]$, which has been run in Matlab. Therefore each iteration corresponds to an analysis frame equal to $\mathrm{n} \times \mathrm{w}_{\text {SIZE }}$; eventually, the last iteration corresponds to a window of $\mathrm{N} \times$ $\mathrm{w}_{\text {SIZE }}$, which is equal to the entire signal length $\mathrm{T}$. Kimura's low runs prediction model is applied at each iteration: as $\mathrm{n}$ increases, the analysed frame corresponds to an amount of data which is $\mathrm{w}_{\text {SIZE }}$ times bigger than the previous one.

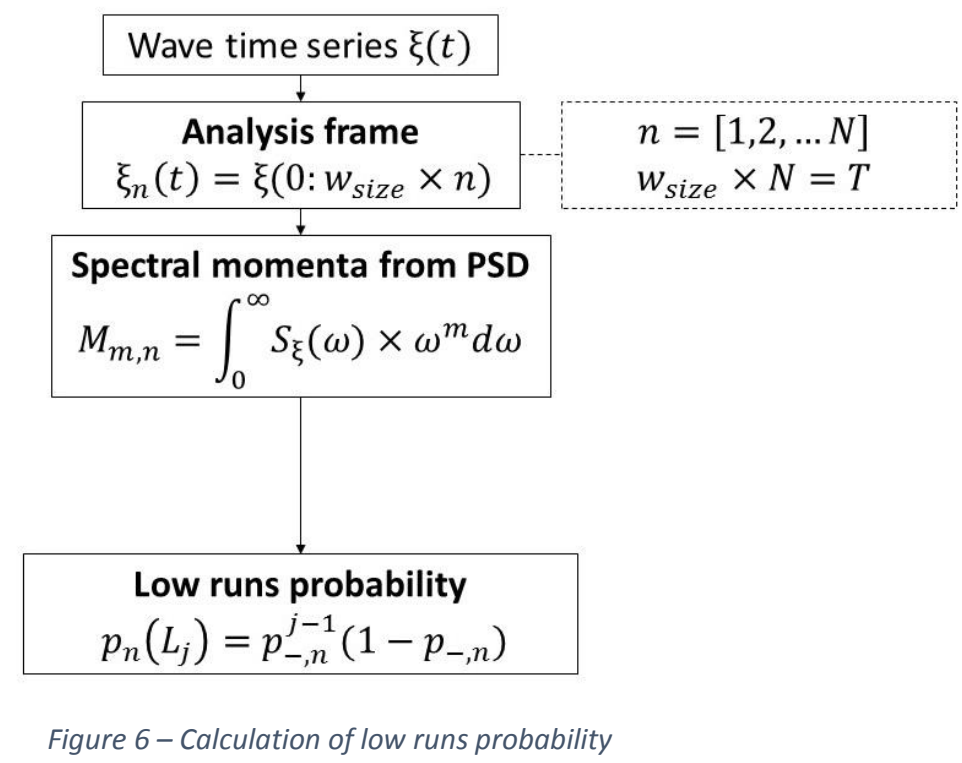

Figs. 7 to 10 report the Kimura's model results for the Channel Coast data, corresponding to June 2017, November 2017, January 2018 and March 2018 respectively: the points in each curve represent the low runs probability corresponding to the current analysis window and its size. For example, the third point from left on the top curve represents the low runs probabilities occurring at the first wave for a window equal to $3 \times \mathrm{w}_{\text {SIZE }}$. In this case, a $\mathrm{w}_{\text {SIZE }}=30 \mathrm{~min}$ has been used, so this point represents the low runs probability for the first wave after 90 minutes of data analysis. Similarly, the fourth point on the second curve from 
the top represents the probability of having a low run at the second incident wave after 120 minutes and so on.

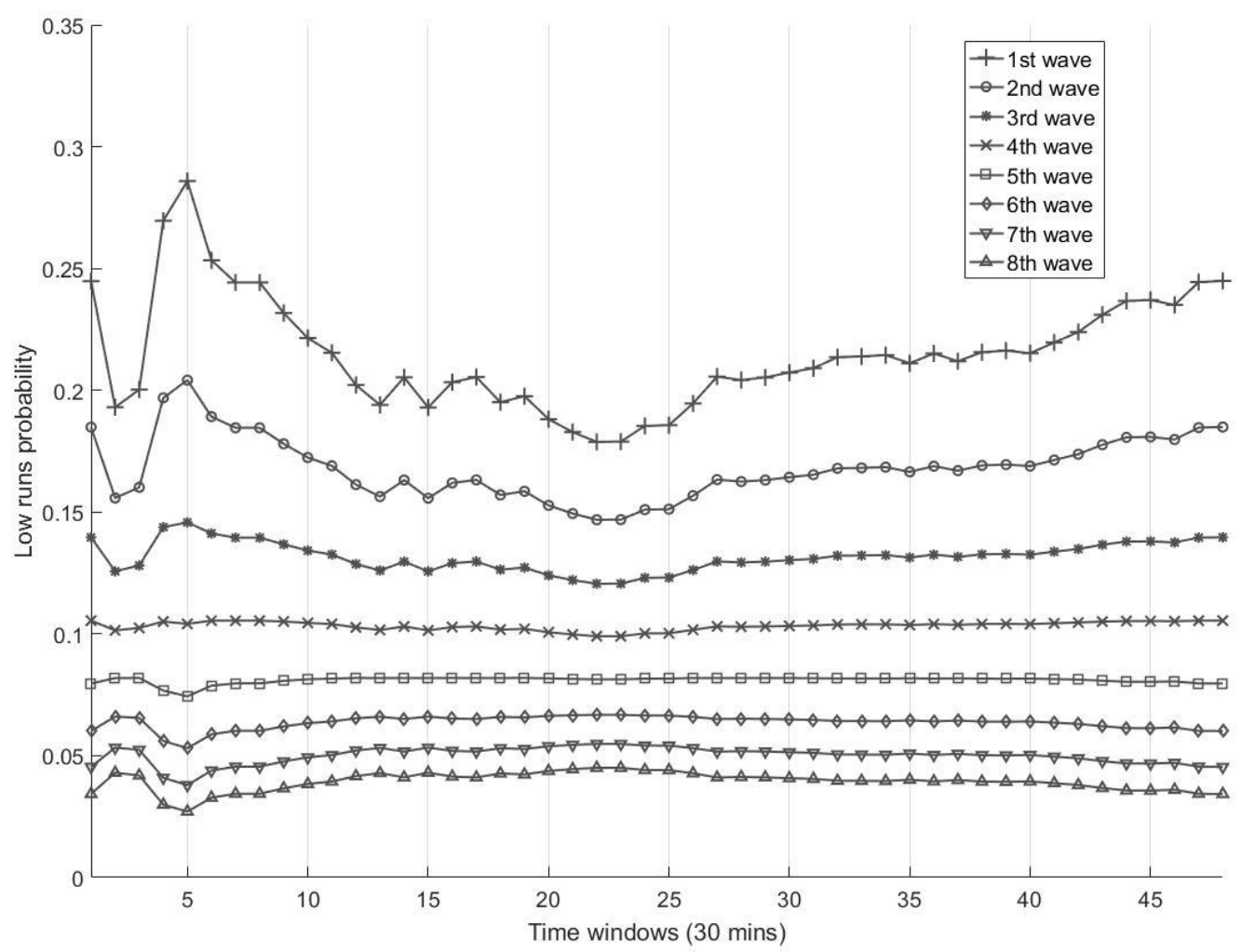

Figure 7- Behaviour of low runs probabilities in time for a $24 \mathrm{~h}$ time series in June 2017 (Channel Coast data) 


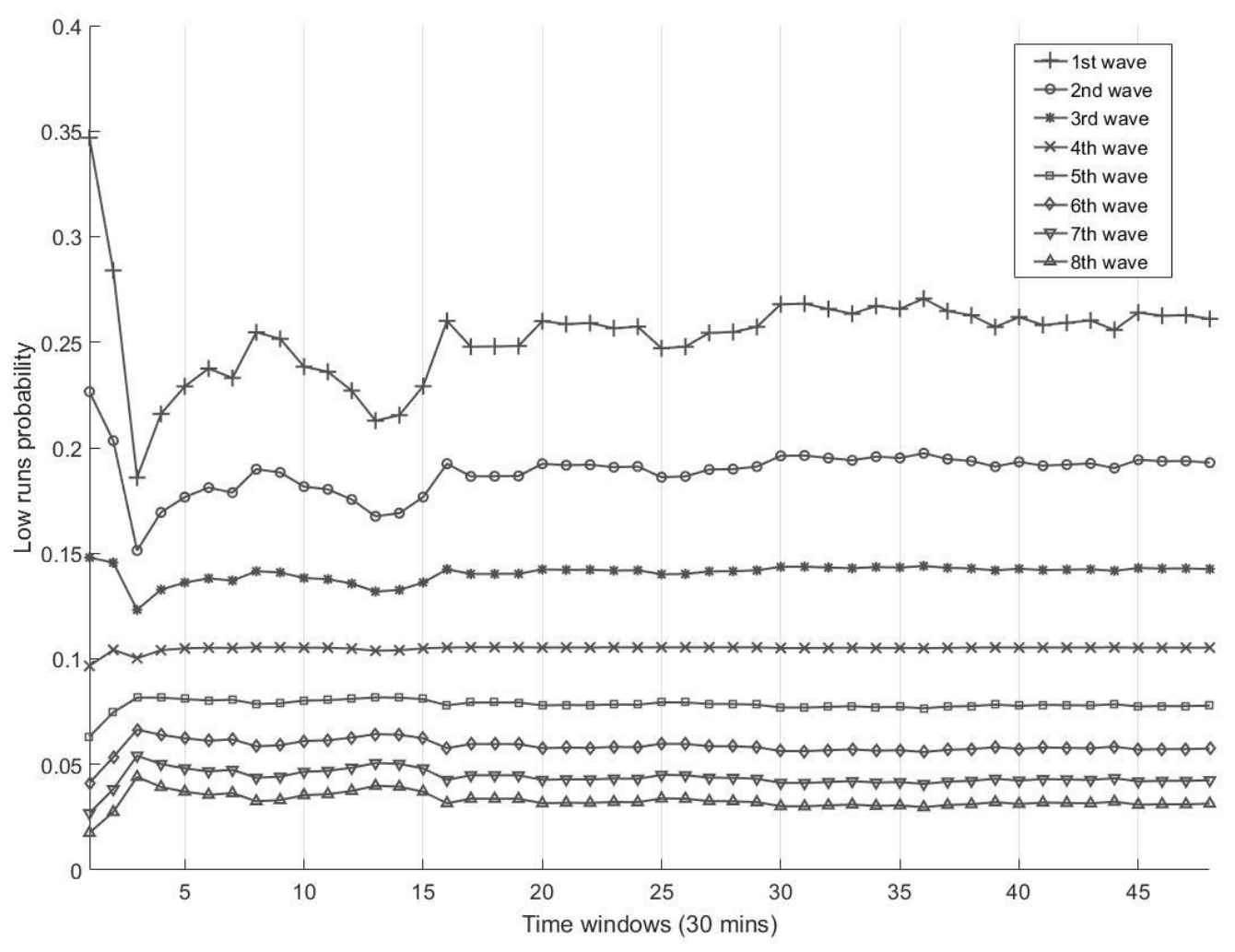

Figure 8- Behaviour of low runs probabilities in time for a $24 \mathrm{~h}$ time series in November 2017 (Channel Coast data) 


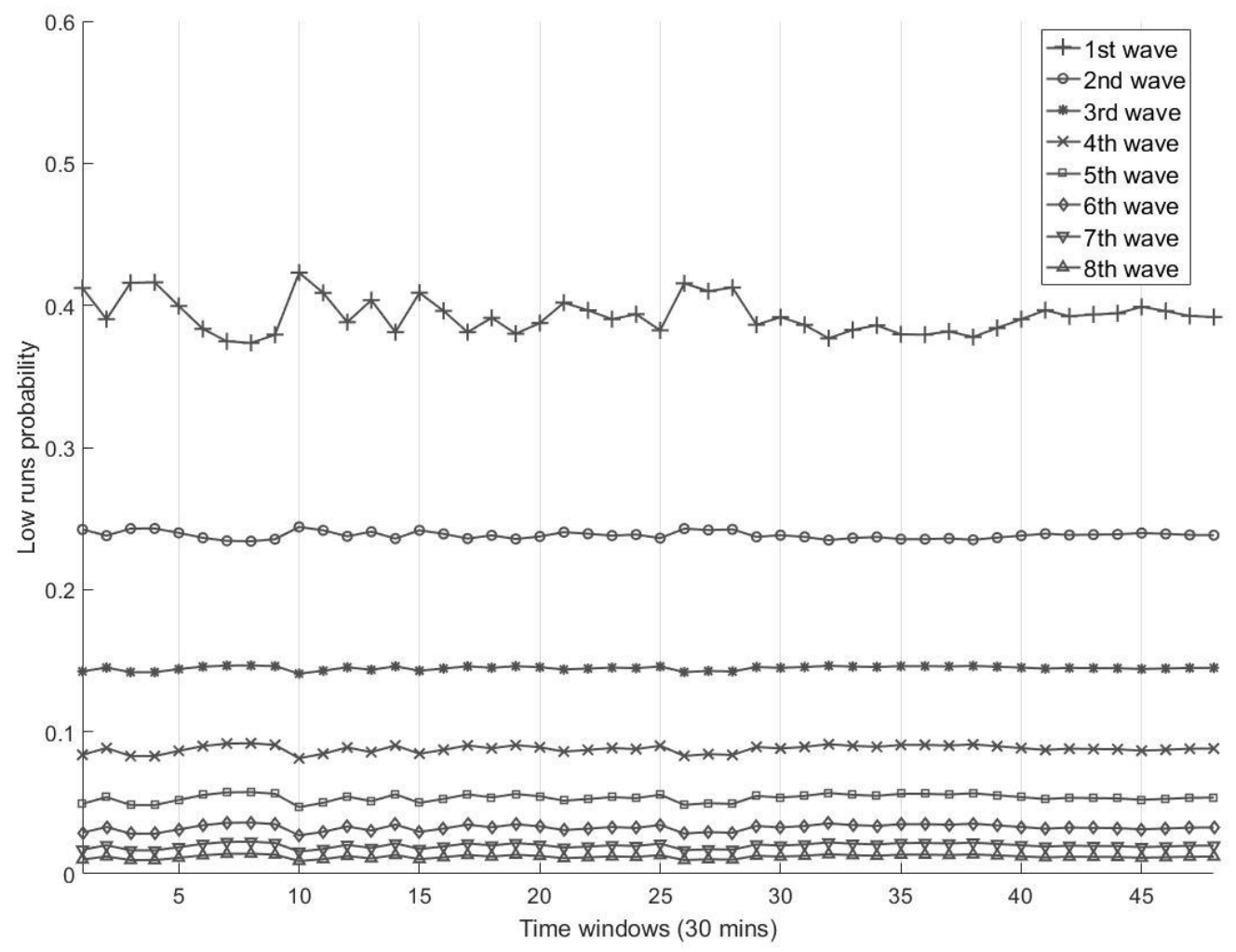

Figure 9- Behaviour of low runs probabilities in time for a $24 \mathrm{~h}$ time series in January 2018 (Channel Coast data) 


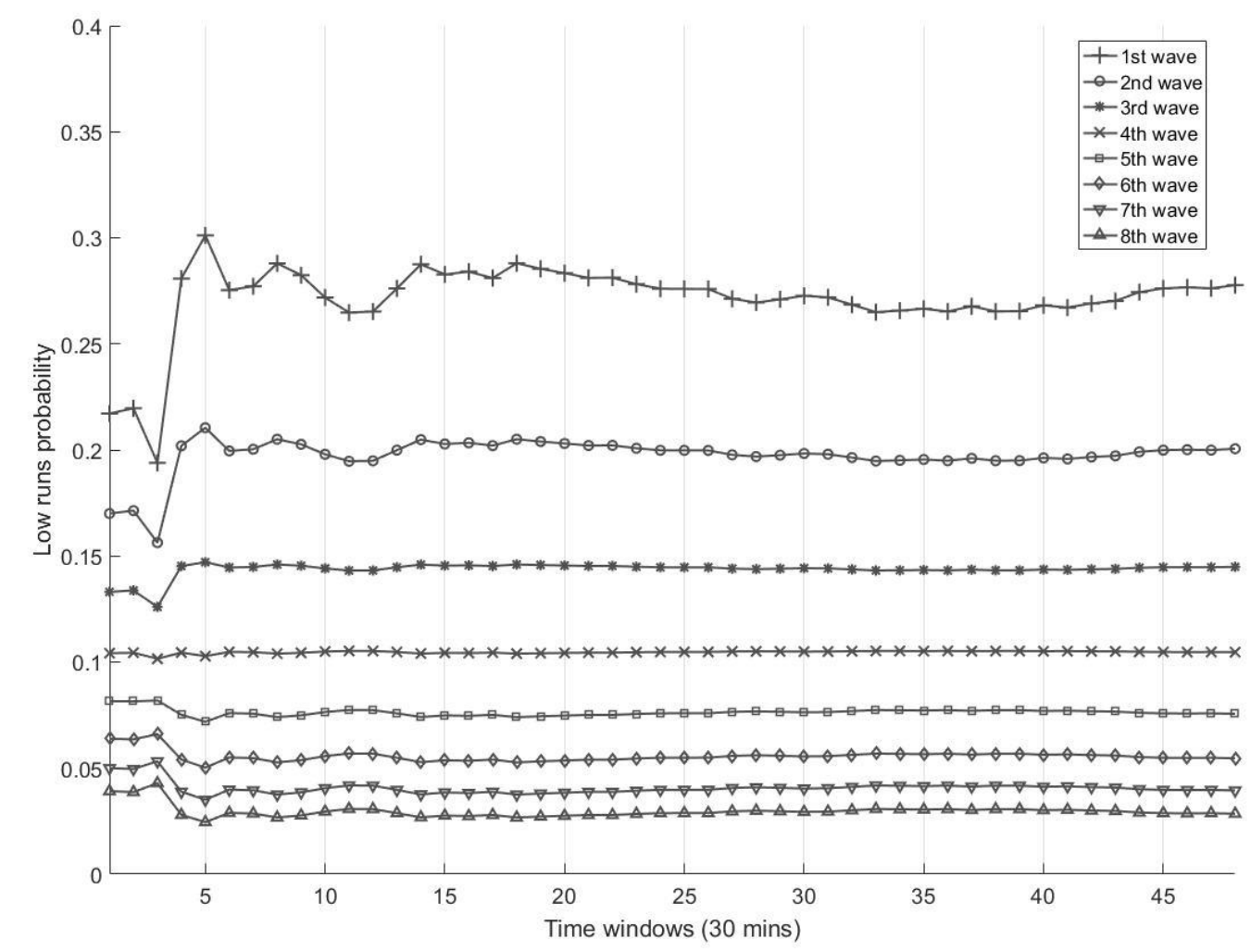

Figure 10- Behaviour of low runs probabilities in time for a 24h time series in March 2018 (Channel Coast data)

An analysis of Fig. 7 to 10 shows that the amount of data required in order to achieve a reliable prediction of the low run probability can change from record to record; the results from the Kimura's method applied to the time series corresponding to June 2017 shows that a sampling time of roughly 15-20 time windows, corresponding to a theoretical amount of 7-8 hours hours which is required to have a stable prediction of the low runs probability. This result should be interpreted by giving a more accurate observation of the behaviour of the probability curves: in the first five windows, the curves shows a quite steep behaviour, to progressively stabilize as more data are elaborated by the model. By observing the maximum and significant wave heights corresponding to June 2017 time series (Fig.5), it is possible to see that they show a quite stable behaviour of the sea conditions. This means that, assuming a sufficiently stable sea condition, the Kimura's model needs a minimum threshold of sample data in order to produce a result that can be considered reliable

Similar results can be observed also for Fig.8, corresponding to November 2017; here, after a steep transient behaviour, the low run probability curves tend to stabilize around the value of 0.25 after 15 time windows, corresponding to 7 hours. Also in this case, by observing the behaviour of the corresponding significant and maximum wave height (Fig.5), it is possible to observe a quite stable conditions of the sea conditions.

For Figures 9 and 10 (November and March 2018) it seems that the required sampling time is slightly shorter (around 10 time windows, corresponding to 3 hours). 
In general, it is possible to conclude that the Kimura's model is characterized by an amount of time that is required to elaborate a record corresponding to a specific sea condition and provide a reliable result for it.

Moreover, it is important to highlight an important aspect about the intrinsic limit of the Kimura's methodology in such contexts: the low runs probability calculated on the base of the recorded data only provides information about the probability of the next run and its average length, however it does not give any indication about the timing at which the low run is expected to occur.

More generally, it is possible to say that Kimura's theory, however valuable, provides estimation on a very low resolution time scale.

\section{Conclusions}

The paper has discussed the limitation of the traditional statistical backwards approach for the prediction of low runs in short time applications, such as launch and recovery operations.

A correlation between weather conditions, spectrum bandwidth and wave to wave correlation has been shown: conditions of rough sea generally correspond to broader spectra and higher values of correlation between two consecutive waves. The effect of spectrum broadness and wave groupiness is remarkably reflected on the low run prediction calculated with the Kimura's theory: more correlated time series correspond to flatter curves in a low runs probability vs. run length diagram (the run length is expressed as number of incoming waves); such conditions correspond to curves which are settled on low probability values (around $20-25 \%$ ), which results in a poor estimation of the length of the next low run.

Moreover, by calculating the low runs probability in an adaptive window that expands along the signal, it is shown that a stable prediction occurs after circa six to eight hours of local data recording in conditions of rough sea.

Eventually, another intrinsic limitation of the statistic estimation of the low runs consists in the fact that it only gives information about the length of the next run, but not on when the next run is expected to happen: such information is vital in the QP prediction realm.

Future investigations on this work involve the development of a wave synthesis methodology in order to produce realistic time series to be arbitrarily used as test data for the statistical runs prediction methodology.

\section{Funding resources}

This research work was supported by the Engineering and Physical Sciences Research Council (EPSRC) (grant number EP/N008707/1)

\section{Acknowledgements}


A special mention goes to Prof. Mike Belmont, Dr. Jacqueline Christmas and Dr. Mustafa AlAni from the University of Exeter for providing help with the Matlab code implementation of the Kimura's model.

\section{Declaration of interest}

None

\section{BIBLIOGRAPHY}

[1] Burmesteir H-C., Bruhn, W., Rødesth, $\varnothing$., J., Porathe, T. , 2014 - Autonomous Unmanned Merchant Vessel and its Contribution towards the e-Navigation Implementation: The MUNIN Perspective - International Journal of e-Navigation and Maritime Economy- 13.

[2] Levander, O. , 2015- Unmanned Ships Älykäs Meriteollisuus - Seminar.

[3] Giron-Sierra, J.,M., Jimenez, J., M. , 2010 - State-of-the-Art of Wave Measurement for Ship Motion Prediction - 8th IFAC Conference on Control Applications in Marine Systems Rostock-Warnemünde, Germany September 15-17.

[4] Nieto Borge, J., C., Rodriguez, G., R., Hessner, K., Gonzalez, P., I. , 2004 - Inversion of Marine Radar Images for Surface Wave Analysis - American Meteorological Society.

[5] Kimura, A. , 1980-Statistical properties of random wave groups - Coastal Engineering.

[6] Elgar, S., Guza, R., T., Seymour, R. J. - Groups of Waves in Shallow Water - Journal Of Geophysical Research, Vol. 89, No. c3, pages 3623-3634.

[7] Saulnier, J., Clément, A., Falcão, A., Pontes, T., Prevosto, M., Ricci, P. , 1984 - Wave groupiness and spectral bandwidth as relevant parameters for the performance assessment of wave energy -Ocean Engineering, Elsevier, pp.130-147, 2011, 38 (1).

[8] Giron-Sierra, J., M., Esteban, S. , 2010 - The Problem of Quiescent Period Prediction for Ships: A Review - 8th IFAC Conference on Control Applications in Marine Systems Rostock Warnemünde, Germany September 15-17.

[9] Edgar, D., R., Horwood, J.M.K., Thurley, R., Belmont, M., R. , 2000 - The effects of parameters on the maximum prediction time possible in short term forecasting of the sea surface shape - Int. Shipbuild. Progr., 47, no. 451 pp. 287-301.

[10] Lloyd, A.,R.,J.,M.,1989 - Seakeeping - Ship behaviour in rough weather .

[11] Cartwright, D. E. \& Longuet-Higgins, M. S. 1956 The statistical distribution of the maxima of a random function. Proc. R. Soc. Lond. A 237, 212-232.

[12] Longuet - Higgins, M., S. , 1984-Statistical properties of wave groups in a random sea state - The Royal Society Publishing - Volume 312, issue 1521.

[13] Uhlenbeck, G.E., 1943. Theory of random process. MIT Radiation Lab, Rep. 454. 
[14] https://www.channelcoast.org - May 2018

[15] https://www.britannica.com/science/Douglas-scale- May 2018 